Japan J. Indust. Appl. Math. manuscript No.

(will be inserted by the editor)

\title{
On the construction of new families of wavelets
}

\author{
Naohiro Fukuda - Tamotu Kinoshita
}

Received: date / Accepted: date

\begin{abstract}
In this paper, we introduce a new kind of wavelet which converges in $L^{q}$ to the Shannon wavelet as the order parameter $n$ increases. In particular, we shall give a symmetric orthogonal scaling function whose time-bandwidth product is near $1 / 2$ and describe some applications.
\end{abstract}

Keywords Shannon wavelet . Haar wavelet · Battle-Lemarié wavelet . time-bandwidth product

\section{Introduction}

It is well known that the limit of the B-spline family is the Gaussian function, which achieves the smallest time-bandwidth product permitted by the uncertainty principle, i.e., 1/2 (see [10] and [12]). The B-spline and the Gaussian function are not orthogonal to their translates. There are various types of orthogonal wavelet with order parameters, e.g., the Battle-Lemarié wavelet, Daubechies wavelet, and Strömberg wavelet (see [1], [2], [9], and [11]). In particular, [7] showed that the Battle-Lemarié wavelet of order $n$ converges to the Shannon wavelet as $n$ tends to infinity. Let us denote the Battle-Lemarié low pass filter, the scaling function, and the wavelet by $m_{n}^{B L}(\xi), \varphi_{n}^{B L}(x)$, and $\psi_{n}^{B L}(x)$, respectively. This family of the Battle-Lemarié wavelet interpolates the (non-smooth) Haar wavelet which has the best localization in time, and the (smooth) Shannon wavelet which has the best localization in frequency. For some applications, the order parameter $n$ enables control of the smoothness and the proportion between the time window and the frequency window.

Naohiro Fukuda

Institute of Mathematics, University of Tsukuba, Tsukuba Ibaraki 305-8571, Japan

E-mail: naohiro-f@math.tsukuba.ac.jp

Tamotu Kinoshita

Institute of Mathematics, University of Tsukuba, Tsukuba Ibaraki 305-8571, Japan

E-mail: kinosita@math.tsukuba.ac.jp 
On the other hand, the Daubechies wavelet of order $n$ does not converge to the Shannon wavelet as $n$ tends to infinity. The asymptotic behavior of Daubechies filters is studied in [6].

First, we shall introduce another family of wavelets interpolating from the Haar wavelet to the Shannon wavelet. In this paper, the low pass filter of the Haar wavelet $m_{1}^{B L}(\xi)$ is denoted also by $m_{1}^{H}(\xi)$ and given by

$$
m_{1}^{H}(\xi)\left(\equiv m_{1}^{B L}(\xi)\right)=e^{-i \xi / 2} \cos \frac{\xi}{2} .
$$

$m_{1}^{H}(\xi)$ is $2 \pi$-periodic due to the multiplication by $e^{-i \xi / 2}$. We immediately see that $m_{1}^{H}(0)=1$ and $\left|m_{1}^{H}(\xi)\right|^{2}+\left|m_{1}^{H}(\xi+\pi)\right|^{2}=1$. Then we also get the Fourier transform of the scaling function $\hat{\varphi}_{1}^{H}(\xi)=\prod_{j=1}^{\infty} e^{-i \xi / 2^{j+1}} \prod_{j=1}^{\infty} \cos \frac{\xi}{2^{j+1}}=$ $e^{-i \xi / 2} \operatorname{sinc} \frac{\xi}{2}$, where sinc $\xi=\frac{\sin \xi}{\xi}$. Now, let us put

$$
\nu_{n}(\xi)=\left\{\begin{array}{cl}
0 & \text { for } \xi<0, \\
p_{n}(\xi) & \text { for } 0 \leq \xi \leq 1, \\
1 & \text { for } \xi>1,
\end{array}\right.
$$

where $p_{n}(\xi)$ is a polynomial of degree $2 n+1$ satisfying $p_{n}(\xi)+p_{n}(1-\xi) \equiv 1$ and $p_{n}(0)=0$, e.g., $\frac{\int_{0}^{x} t^{n}(1-t)^{n} d t}{\int_{0}^{1} t^{n}(1-t)^{n} d t}$. Then, by neglecting $e^{-i \xi / 2}$ and replacing $\frac{\xi}{2}$ by $\frac{\pi}{2} \nu_{n}\left(\frac{3}{\pi}|\xi|-1\right)$ in the argument of the cosine term of $m_{1}^{H}(\xi)$, one gets

$$
m_{n}^{M}(\xi)=\cos \left(\frac{\pi}{2} \nu_{n}\left(\frac{3}{\pi}|\xi|-1\right)\right) .
$$

The Meyer wavelet family is constructed from $m_{n}^{M}(\xi)(n \geq 1)$, and its Fourier transform belongs to $C^{n}$ due to the irregularity of (1) at the points $\xi=0,1$. This causes polynomial decay of the Meyer wavelet at $\infty$.

Remark 1 One can find a Gevrey function $p_{\infty}$ such that $\nu_{\infty}$ is also a Gevrey function. Then, the Meyer wavelet would have arbitrary polynomial decay. However, there does not exist any analytic function $p_{\infty}$ such that $\nu_{\infty}$ is also analytic. Therefore in this construction with (1), it is impossible to have exponential decay.

In the present paper, we shall replace $\frac{\xi}{2}$ by $\frac{\pi}{2} \sin ^{2} \frac{\xi}{2}$ in the argument of the cosine term of $m_{1}^{H}(\xi)$ and define

$$
m_{2}^{H}(\xi)=\cos \left(\frac{\pi}{2} \sin ^{2} \frac{\xi}{2}\right) .
$$

Here we remark that $m_{2}^{H}(\xi)$ is $2 \pi$-periodic and satisfies $m_{2}^{H}(0)=1$ and $\left|m_{2}^{H}(\xi)\right|^{2}+\left|m_{2}^{H}(\xi+\pi)\right|^{2}=1$, since

$$
m_{2}^{H}(\xi+\pi)=\cos \left(\frac{\pi}{2} \cos ^{2} \frac{\xi}{2}\right)=\cos \left(\frac{\pi}{2}-\frac{\pi}{2} \sin ^{2} \frac{\xi}{2}\right)=\sin \left(\frac{\pi}{2} \sin ^{2} \frac{\xi}{2}\right) .
$$

To construct a new wavelet family, let us consider $\Theta_{n}(\xi)$ given recursively by

$$
\Theta_{1}(\xi)=\frac{\xi}{2} \text { and } \Theta_{n}(\xi)=\frac{\pi}{2} \sin ^{2} \Theta_{n-1}(\xi) \text { for } n \geq 2 .
$$


Remark 2 For $n \geq 1$ we see that $\Theta_{n}(0)=0, \Theta_{n}\left(\frac{\pi}{2}\right)=\frac{\pi}{4}$ and $\Theta_{n}(\pi)=\frac{\pi}{2}$ hold.

We also define the $2 \pi$-periodic function

$$
m_{n}^{H}(\xi)=\cos \Theta_{n}(\xi) \text { for } n \geq 2 .
$$

$m_{n}^{H}(\xi)$ satisfies $m_{n}^{H}(0)=1$. Noting that $m_{n}^{H}(\xi+\pi)=\sin \Theta_{n}(\xi)$ still holds, we can obtain $\left|m_{n}^{H}(\xi)\right|^{2}+\left|m_{n}^{H}(\xi+\pi)\right|^{2}=1$. Therefore, since clearly $m_{n}^{H}$ is differentiable, we find that $\prod_{j=1}^{\infty} m_{n}^{H}\left(2^{-j} \xi\right)$ converges uniformly on bounded sets of $\mathbf{R}$ (see [5]). Thus, we can define $\varphi_{n}^{H}(x)$ by

$$
\hat{\varphi}_{n}^{H}(\xi)=\prod_{j=1}^{\infty} m_{n}^{H}\left(2^{-j} \xi\right) .
$$

\section{Main Results}

Denoting as $m_{\infty}^{S H}(\xi)$ the low pass filter of the Shannon wavelet, i.e., the $2 \pi$ periodic function defined by

$$
m_{\infty}^{S H}(\xi)= \begin{cases}1 & \text { for }|\xi| \leq \frac{\pi}{2} \\ 0 & \text { for } \frac{\pi}{2}<|\xi| \leq \pi\end{cases}
$$

we get the following properties for $m_{n}^{H}(\xi)$, defined in (3):

Proposition $1 m_{n}^{H}(\xi)$ satisfies that for all $n \geq 1$,

$$
m_{n}^{H}(\xi) \neq 0 \text { for }|\xi| \leq \frac{\pi}{2}
$$

and

$$
\lim _{n \rightarrow \infty} m_{n}^{H}(\xi)=m_{\infty}^{S H}(\xi) \text { for } \xi \in \mathbf{R} \backslash\left\{\pi \mathbf{Z}+\frac{\pi}{2}\right\}
$$

By (5), it follows that $m_{n}^{H}(\xi)$ is the low pass filter for an MRA (see [5]). This means that $\psi_{n}^{H}(x)$ can be defined by its Fourier transform

$$
\hat{\psi}_{n}^{H}(\xi)=e^{i \xi / 2} \overline{m_{n}^{H}\left(\frac{\xi}{2}+\pi\right)} \hat{\varphi}_{n}^{H}\left(\frac{\xi}{2}\right) .
$$

By (6), the scaling function $\varphi_{n}^{H}$ and wavelet $\psi_{n}^{H}$ also converge to the Shannon scaling function $\varphi_{\infty}^{S H}$ and wavelet $\psi_{\infty}^{S H}$ as the order parameter $n$ increases. More precisely, we can prove the following:

Theorem 1 For $2 \leq q \leq \infty$, we have

$$
\lim _{n \rightarrow \infty}\left\|\varphi_{n}^{H}-\varphi_{\infty}^{S H}\right\|_{L^{q}}=0 \text { and } \lim _{n \rightarrow \infty}\left\|\psi_{n}^{H}-\psi_{\infty}^{S H}\right\|_{L^{q}}=0
$$




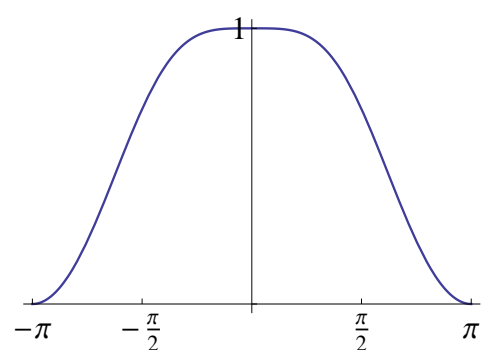

(a) $m_{2}^{H}$

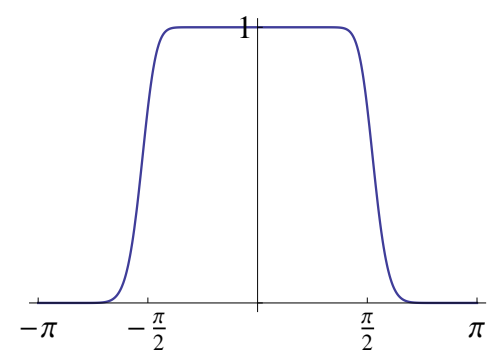

(c) $m_{5}^{H}$

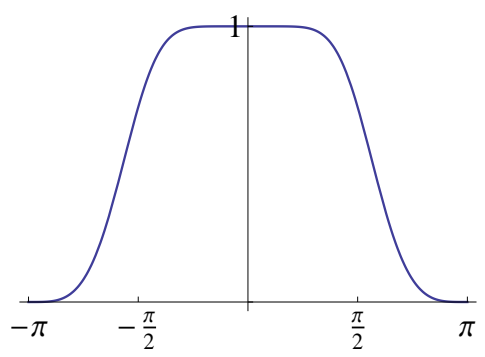

(b) $m_{3}^{H}$

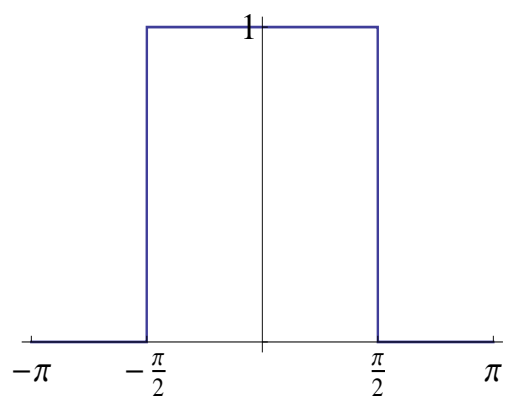

(d) $m_{\infty}^{S H}$

Fig. 1 Graphs of $m_{2}^{H}, m_{3}^{H}, m_{5}^{H}$, and $m_{\infty}^{S H}$.

The proofs of Proposition 1 and Theorem 1 will be given in $\S 4$ and $\S 5$, respectively. In [7] one can see the corresponding results for the Battle-Lemarié scaling function and wavelet.

$\varphi_{\infty}^{S H}$ and $\psi_{\infty}^{S H}$ have polynomial decay and belong to $C^{\infty}\left(\mathbf{R}_{x}\right)$, since $\varphi_{\infty}^{S H}(x)=$ $\operatorname{sinc}(\pi x)$ and $\psi_{\infty}^{S H}(x)=2 \operatorname{sinc}(2 \pi x)-\operatorname{sinc}(\pi x)$. In particular, for case $n=2$, $\hat{\varphi}_{n}^{H}$ can be rewritten as

$$
\begin{aligned}
\hat{\varphi}_{2}^{H}(\xi) & =\prod_{j=1}^{\infty} \cos \left(\frac{\pi}{2} \sin ^{2}\left(\frac{\xi}{2^{j+1}}\right)\right)=\prod_{j=1}^{\infty} \sin \left(\frac{\pi}{2} \cos ^{2}\left(\frac{\xi}{2^{j+1}}\right)\right) \\
& =\prod_{j=1}^{\infty} \cos ^{2}\left(\frac{\xi}{2^{j+1}}\right) \prod_{j=1}^{\infty}\left\{\frac{\pi}{2} \operatorname{sinc}\left(\frac{\pi}{2} \cos ^{2}\left(\frac{\xi}{2^{j+1}}\right)\right)\right\} \\
& =\operatorname{sinc}^{2} \frac{\xi}{2} \prod_{j=1}^{\infty} L\left(\frac{\xi}{2^{j}}\right),
\end{aligned}
$$

where $L(\xi)=\frac{\pi}{2} \operatorname{sinc}\left(\frac{\pi}{2} \cos ^{2} \frac{\xi}{2}\right)$. We remark that $L(\xi+i \eta)$ is an entire function satisfying $L(0)=1$. By continuity, there exists $C>0$ such that

$$
|L(\xi+i \eta)| \leq 1+C|\xi+i \eta| \text { in some neighborhood of the origin. }
$$


Since $|L(\xi)| \leq \frac{\pi}{2}$ for $\xi \in \mathbf{R}$, for all $\varepsilon>0$ there exists $\delta_{\xi}>0$ such that for all $\eta$ with $0<|\eta|<\delta_{\xi}$, we have

$$
|L(\xi+i \eta)| \leq\left(\frac{\pi}{2}\right)^{1+\varepsilon} .
$$

We note that $L(\xi)$ is $2 \pi$-periodic and $\sup _{\xi \in \mathbf{R}} \delta_{\xi}=\max _{0 \leq \xi \leq 2 \pi} \delta_{\xi}>0$. So, we can take $\delta=\max _{0 \leq \xi \leq 2 \pi} \delta_{\xi}>0$ which is independent of $\xi$. For arbitrarily fixed $J>0$, taking $\eta$ such that $\max _{1 \leq j \leq J}\left|\frac{\eta}{2^{j}}\right|<\delta$, i.e., $|\eta|<2 \delta$, by (7) and (8), we get for $2^{J} \leq|\xi+i \eta| \leq 2^{J+1}$

$$
\begin{aligned}
\left|\hat{\varphi}_{2}^{H}(\xi+i \eta)\right| & =\left|\operatorname{sinc}\left(\frac{\xi+i \eta}{2}\right)\right|^{2} \prod_{j=1}^{J}\left|L\left(\frac{\xi+i \eta}{2^{j}}\right)\right| \prod_{j=J+1}^{\infty}\left|L\left(\frac{\xi+i \eta}{2^{j}}\right)\right| \\
& \leq\left|\frac{\sin \left(\frac{\xi+i \eta}{2}\right)}{\left(\frac{\xi+i \eta}{2}\right)}\right|^{2}\left(\frac{\pi}{2}\right)^{J(1+\varepsilon)} \prod_{j=J+1}^{\infty}\left\{1+C\left|\frac{\xi+i \eta}{2^{j}}\right|\right\} \\
& \leq \frac{C_{\eta}}{|\xi+i \eta|^{2}+1} 2^{J(1+\varepsilon) \log _{2} \frac{\pi}{2}} \prod_{j=J+1}^{\infty} \exp \left\{C\left|\frac{\xi+i \eta}{2^{j}}\right|\right\} \\
& \leq \frac{C_{\eta}}{|\xi+i \eta|^{2}+1}|\xi+i \eta|^{(1+\varepsilon) \log _{2} \frac{\pi}{2}} \exp \left\{\sum_{j=0}^{\infty} \frac{C}{2^{j}}\right\} \\
& \leq C_{\eta} e^{2 C}(|\xi|+|\eta|+1)^{q_{\varepsilon}}
\end{aligned}
$$

where $q_{\varepsilon}=(1+\varepsilon) \log _{2} \frac{\pi}{2}-2$ and $C_{\eta}=4 \cosh \eta$, since $\left|\sin \left(\frac{\xi+i \eta}{2}\right)\right|^{2}=$ $\left|\sin \left(\frac{\xi}{2}\right) \cosh \left(\frac{\eta}{2}\right)+i \cos \left(\frac{\xi}{2}\right) \sinh \left(\frac{\eta}{2}\right)\right|^{2} \leq \cosh ^{2}\left(\frac{\eta}{2}\right)+\sinh ^{2}\left(\frac{\eta}{2}\right)=\cosh \eta$. For $|\xi+i \eta| \leq 1$, we easily see that $\left|\hat{\varphi}_{2}^{H}(\xi+i \eta)\right| \leq C^{\prime}$. Thus, it follows that for $\xi \in \mathbf{R}$ and $|\eta|<2 \delta$

$$
\left|\hat{\varphi}_{2}^{H}(\xi+i \eta)\right| \leq M_{\eta}(|\xi|+|\eta|+1)^{q_{\varepsilon}},
$$

where $M_{\eta}=C_{\eta} e^{2 C}+C^{\prime}$. The exponent $q_{\varepsilon}$ becomes negative for a sufficiently small $\varepsilon>0$. Thus, by the Paley-Wiener theorem, we conclude the following two facts (see $[8]$ ):

- $\varphi_{2}^{H}$ has exponential decay, since $\hat{\varphi}_{2}^{H}$ is analytic in a strip $\{\xi+i \eta:|\eta|<\tilde{\delta}\}$ for some $\tilde{\delta}$.

- $\varphi_{2}^{H}$ belongs to $C^{\alpha_{2}}\left(\mathbf{R}_{x}\right)$ for some $\alpha_{2}>0$ (the estimate (9) with $\eta=0$ ).

Remark 3 Since $m_{n}^{H} \in C^{\infty}$, we can at least expect that $\varphi_{n}^{H}$ has arbitrary polynomial decay for all $n \geq 2$.

Moreover, we can know the decays and regularities of $\varphi_{n}^{H}$ and $\psi_{n}^{H}(n \geq 2)$ as follows: 
Theorem 2 Let $n \geq 2$. The scaling function $\varphi_{n}^{H}$ and wavelet $\psi_{n}^{H}$ have exponential decays and belong to $C^{\alpha_{n}}\left(\mathbf{R}_{x}\right)$ for some $\alpha_{n}>0$ increasing in the parameter $n$.

Above, we proved Theorem 2 only for $n=2$. The case of $n \geq 2$ will be proved in $\S 7$. In fact, we obtain a more refined estimate than (9) in $\S 6$ and a better $\alpha_{n}>0$ is given in Table 1. We also give the time-bandwidth product $\Delta_{f} \Delta_{\hat{f}}$ of the scaling function and wavelet in Table 2 , where $\Delta_{f}$ is defined by

$$
\Delta_{f}:=\left\{\frac{\int_{-\infty}^{\infty}\left(x-x_{0}\right)^{2}|f(x)|^{2} d x}{\int_{-\infty}^{\infty}|f(x)|^{2} d x}\right\}^{1 / 2} \text { with } x_{0}:=\frac{\int_{-\infty}^{\infty} x|f(x)|^{2} d x}{\int_{-\infty}^{\infty}|f(x)|^{2} d x} .
$$

By the uncertainty principle, the lower bound is $1 / 2 . \Delta_{\varphi_{3}^{H}} \Delta_{\hat{\varphi}_{3}^{H}}$ seems very good compared with some other famous scaling functions, given in Table 3.

Table 1 Regularities of $\varphi_{n}^{H}$ and $\psi_{n}^{H}$.

\begin{tabular}{|r|c|c|c|c|c|}
\hline$n$ & 2 & 3 & 4 & 5 & 6 \\
\hline$\alpha_{n}$ & 0.386 & 1.133 & 2.616 & 5.580 & 11.508 \\
\hline
\end{tabular}

Table 2 Time-bandwidth products of the scaling function and wavelet.

\begin{tabular}{|c|c|c|c|c|c|}
\hline$n$ & 2 & 3 & 4 & 5 & 6 \\
\hline$\Delta_{\varphi_{n}^{H}} \Delta_{\hat{\varphi}_{n}^{H}}$ & 0.926 & $\mathbf{0 . 6 6 9}$ & 0.772 & 0.947 & 1.177 \\
\hline$\Delta_{\psi_{n}^{H}} \Delta_{\hat{\psi}_{n}^{H}}$ & 2.603 & 2.136 & 2.500 & 3.069 & 5.393 \\
\hline
\end{tabular}

Table 3 Time-bandwidth products of the Battle-Lemarié, Meyer, and Daubechies scaling functions.

\begin{tabular}{|c|c|c|c|c|c|}
\hline$n$ & 1 & 2 & 3 & 4 & 5 \\
\hline$\Delta_{\varphi_{n}^{B L}} \Delta_{\hat{\varphi}_{n}^{B L}}$ & $\infty$ & 0.686 & 0.741 & 0.837 & 0.928 \\
\hline$\Delta_{\varphi_{n}^{M}} \Delta_{\hat{\varphi}_{n}^{M}}$ & 0.810 & 0.875 & 0.949 & 1.012 & 1.065 \\
\hline$\Delta_{\varphi_{n}^{D}} \Delta_{\hat{\varphi}_{n}^{D}}$ & $\infty$ & 1.057 & 0.828 & 0.849 & 0.984 \\
\hline
\end{tabular}

In conclusion, we observe that $\varphi(x)$ defined by

$$
\hat{\varphi}(\xi)=\prod_{j=1}^{\infty} \cos \left(\frac{\pi}{2} \sin ^{2}\left(\frac{\pi}{2} \sin ^{2} \frac{\xi}{2^{j+1}}\right)\right)
$$

is differentiable in $x$ and satisfies $\Delta_{\varphi} \Delta_{\hat{\varphi}}=0.669$ which is close to $1 / 2$.

\section{Some Generalizations}

In the previous section, starting from the Haar wavelet and using the recursion (2), we constructed a new family of wavelets. A similar procedure can also be carried out for other orthonormal wavelets. For instance, we shall start from the Franklin wavelet as follows: 


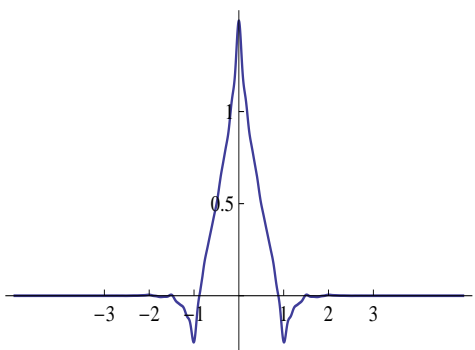

(a) $\varphi_{2}^{H}$

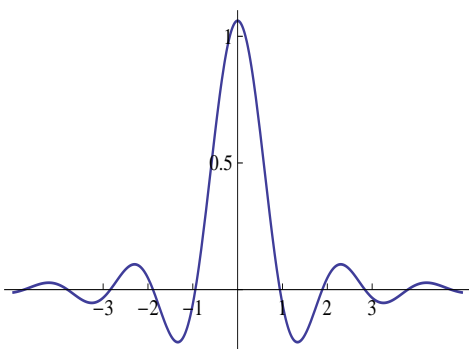

(c) $\varphi_{5}^{H}$

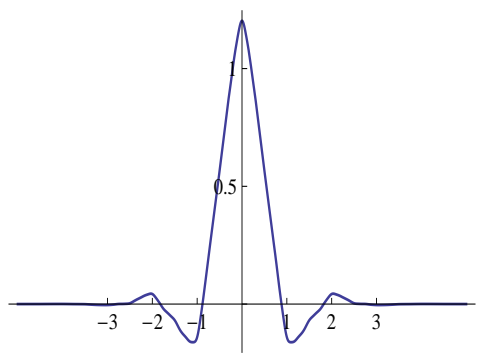

(b) $\varphi_{3}^{H}$

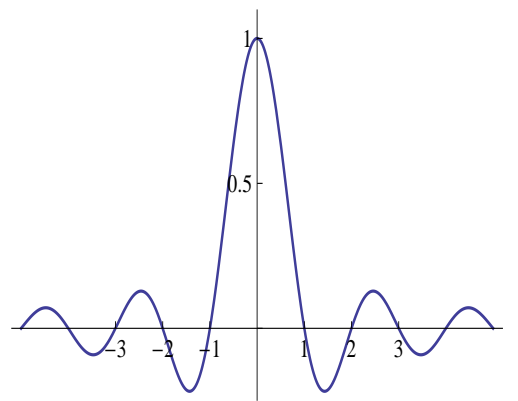

(d) $\varphi_{\infty}^{S H}$

Fig. 2 Graphs of $\varphi_{2}^{H}, \varphi_{3}^{H}, \varphi_{5}^{H}$ and $\varphi_{\infty}^{S H}$.

1. The low pass filter of the Franklin wavelet $m_{2}^{B L}(\xi)$ is also denoted by $m_{1}^{F}(\xi)$ and given by

$$
m_{1}^{F}(\xi)\left(\equiv m_{2}^{B L}(\xi)\right)=\cos ^{2} \frac{\xi}{2} \sqrt{\frac{2 \cos ^{2} \frac{\xi}{2}+1}{8 \cos ^{4} \frac{\xi}{2}-8 \cos ^{2} \frac{\xi}{2}+3}} .
$$

2. We replace $\frac{\xi}{2}$ by $\Theta_{n}(\xi)$ and define

$$
\begin{aligned}
m_{n}^{F}(\xi) & =\cos ^{2} \Theta_{n}(\xi) \sqrt{\frac{2 \cos ^{2} \Theta_{n}(\xi)+1}{8 \cos ^{4} \Theta_{n}(\xi)-8 \cos ^{2} \Theta_{n}(\xi)+3}} \\
& =\cos ^{2} \Theta_{n}(\xi) \sqrt{\frac{2+\cos \left(2 \Theta_{n}(\xi)\right)}{2+\cos \left(4 \Theta_{n}(\xi)\right)}} .
\end{aligned}
$$

3. The scaling function $\varphi_{n}^{F}(x)$ and wavelet $\psi_{n}^{F}(x)$ are defined by

$$
\hat{\varphi}_{n}^{F}(\xi)=\prod_{j=1}^{\infty} m_{n}^{F}\left(2^{-j} \xi\right) \text { and } \hat{\psi}_{n}^{F}(\xi)=e^{i \xi / 2} \overline{m_{n}^{F}\left(\frac{\xi}{2}+\pi\right)} \hat{\varphi}_{n}^{F}\left(\frac{\xi}{2}\right)
$$

More generally, we know the following: 
Proposition 2 Let $\psi$ be an MRA wavelet. Suppose that a low pass filter $m$ associated with the scaling function $\varphi$ has real Fourier coefficients. If $|\varphi(x)|$, $|\psi(x)| \leq C(1+|x|)^{-1-\varepsilon}$, then we have the following two facts:

(i) $m_{n}$ defined by

$$
m_{n}(\xi)=m\left(2 \Theta_{n}(\xi)\right)
$$

satisfies $m_{n}(0)=1$ and $\left|m_{n}(\xi)\right|^{2}+\left|m_{n}(\xi+\pi)\right|^{2}=1$.

(ii) $\varphi_{n}(x)$ defined by $\hat{\varphi}_{n}(\xi)=\prod_{j=1}^{\infty} m_{n}\left(2^{-j} \xi\right)$ belongs to $C^{\gamma_{n}}$ for some $\gamma_{n}$ increasing in the parameter $n$.

Hence, we can construct new families by changing the starting wavelets (e.g. the Daubechies wavelet, Strömberg wavelet, etc.).

Remark 4 If we started from the Shannon wavelet, we would have $m_{n}^{S H}(\xi) \equiv$ $m_{\infty}^{S H}(\xi), \varphi_{n}^{S H}(x) \equiv \varphi_{\infty}^{S H}(x)$ and $\psi_{n}^{S H}(x) \equiv \psi_{\infty}^{S H}(x)$ for all $n \geq 1$.

As for the family constructed from the Franklin wavelet, we also get the following:

Theorem 3 For $2 \leq q \leq \infty$,

$$
\lim _{n \rightarrow \infty}\left\|\varphi_{n}^{F}-\varphi_{\infty}^{S H}\right\|_{L^{q}}=0 \text { and } \lim _{n \rightarrow \infty}\left\|\psi_{n}^{F}-\psi_{\infty}^{S H}\right\|_{L^{q}}=0 .
$$

Theorem 4 Let $n \geq 2$. The scaling function $\varphi_{n}^{F}$ and wavelet $\psi_{n}^{F}$ have exponential decays and belong to $C^{\beta_{n}}\left(\mathbf{R}_{x}\right)$ for some $\beta_{n}>0$ increasing in the parameter $n$.

Table 4 Regularities of $\varphi_{n}^{F}$ and $\psi_{n}^{F}$.

\begin{tabular}{|c|c|c|c|c|c|}
\hline$n$ & 2 & 3 & 4 & 5 & 6 \\
\hline$\beta_{n}$ & 1.175 & 2.569 & 5.358 & 10.934 & 22.086 \\
\hline
\end{tabular}

Proposition 2 will be proved in $\S 7$, and a sketch of the proofs of Theorems 3 and 4 will be given in $\S 8$. Furthermore, we shall present a full generalization of our construction. For this purpose, let us assume that low pass filters have real Fourier coefficients as in Proposition 2. We have constructed families of Haar type and Franklin type by composing $2 \Theta_{n}(\xi)$ with low pass filters. In the substitution for $2 \Theta_{n}(\xi)$, we may also use a real-valued function $\mu(\xi)$ such that

1. $\mu(0)=0, \mu\left(\frac{\pi}{2}\right)=\frac{\pi}{2}$, and $\mu(\pi)=\pi$.

2. $0<\mu(\xi)<\xi$ for $0<\xi<\frac{\pi}{2}$ and $\xi<\mu(\xi)<\pi$ for $\frac{\pi}{2}<\xi<\pi$.

3. $\mu(\xi)+\mu(\xi+\pi)=\pi$. 
A typical example of $\mu$ is given by $\pi\left|m_{0}(\xi+\pi)\right|^{2}$ with a low pass filter $m_{0}$. Then, with another low pass filter $m$, we consider the function $m(\mu(\xi))$, which is $2 \pi$-periodic and satisfies $m(\mu(0))=m(0)=0$. Moreover, we can easily verify that $|m(\mu(\xi))|^{2}+|m(\mu(\xi+\pi))|^{2}=\pi$ in the same way as will be shown in $\S 7$. Thus, the function $m(\mu(\xi))$ is also a low pass filter. So, it is possible to produce other types of filters by mixing some $\mu$ 's.

Example 1 The original Haar filter $m^{H}(\xi)$ is $e^{-i \frac{\xi}{2}} \cos \frac{\xi}{2}$. Let us denote the (modified) Haar-type filter $\cos \xi / 2$ by $m^{\tilde{H}}(\xi)$, where the multiplication by $e^{-i \xi / 2}$ has been neglected as in $\S 1$. The functions $\mu$ 's corresponding to Haar, Franklin, and Daubechies are defined respectively by

$$
\begin{aligned}
& \mu_{\tilde{H}}(\xi)\left(=\mu_{H}(\xi)\right)=\pi\left|m^{\tilde{H}}(\xi+\pi)\right|^{2}=\pi \sin ^{2} \frac{\xi}{2}, \\
& \mu_{F}(\xi)=\pi\left|m^{F}(\xi+\pi)\right|^{2}=\pi \sin ^{4}\left(\frac{\xi}{2}\right) \frac{2-\cos \xi}{2+\cos 2 \xi}, \\
& \mu_{D}(\xi)=\pi\left|m^{D}(\xi+\pi)\right|^{2}=\pi \sin ^{4}\left(\frac{\xi}{2}\right)(2+\cos \xi) .
\end{aligned}
$$

Using these various $\mu$ 's, we are able to produce other types of scaling functions, for instance,

$$
\begin{gathered}
\hat{\varphi}_{D}^{\tilde{H}}(\xi)=\prod_{j=1}^{\infty} m_{D}^{\tilde{H}}\left(2^{-j} \xi\right) \quad \text { with } \quad m_{D}^{\tilde{H}}(\xi)=m^{\tilde{H}}\left(\mu_{D}(\xi)\right), \\
\hat{\varphi}_{D D}^{F}(\xi)=\prod_{j=1}^{\infty} m_{D D}^{F}\left(2^{-j} \xi\right) \quad \text { with } \quad m_{D D}^{F}(\xi)=m^{F}\left(\mu_{D}\left(\mu_{D}(\xi)\right)\right), \\
\hat{\varphi}_{D \tilde{H} F}^{\tilde{H}}(\xi)=\prod_{j=1}^{\infty} m_{D \tilde{H} F}^{\tilde{H}}\left(2^{-j} \xi\right) \text { with } m_{D \tilde{H} F}^{\tilde{H}}(\xi)=m^{\tilde{H}}\left(\mu_{D}\left(\mu_{\tilde{H}}\left(\mu_{F}(\xi)\right)\right)\right) .
\end{gathered}
$$

Remark 5 For $\mu_{D}$ and $\mu_{H}$, we have two filters $m_{D \tilde{H}}(\xi)=m\left(\mu_{D}\left(\mu_{\tilde{H}}(\xi)\right)\right)$ and $m_{\tilde{H} D}(\xi)=m\left(\mu_{\tilde{H}}\left(\mu_{D}(\xi)\right)\right)$ with some filter $m$. In general, $\mu_{D}$ and $\mu_{\tilde{H}}$ are not commutable, that is, $m_{D \tilde{H}} \neq m_{\tilde{H} D}$.

We note that $m^{\tilde{H}}$ itself is not a low pass filter, since $m^{\tilde{H}}$ is not $2 \pi$-periodic. But, $m_{D}^{\tilde{H}}$ is $2 \pi$-periodic and becomes a low pass filter thanks to composition with $\mu_{D}(\xi)$. Using the above notation, we can also denote $\varphi_{3}^{H}$ in $\S 1$ by $\varphi_{\tilde{H} \tilde{H}}^{\tilde{H}}$, whose time-bandwidth product is 0.669 . In particular, we observe that $\varphi_{D}^{\tilde{H}}$ defined by

$$
\hat{\varphi}_{D}^{\tilde{H}}(\xi)=\prod_{j=1}^{\infty} \cos \left(\frac{\pi}{2} \sin ^{4} \frac{\xi}{2^{j+1}}\left(2+\cos \frac{\xi}{2^{j}}\right)\right)
$$

is differentiable and has exponential decay, and that its time-bandwidth product is 0.667 , which is the best result as far as we know. We also remark that 


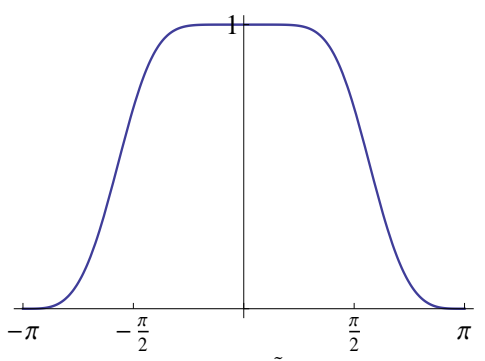

(a) $m_{D}^{\tilde{H}}$

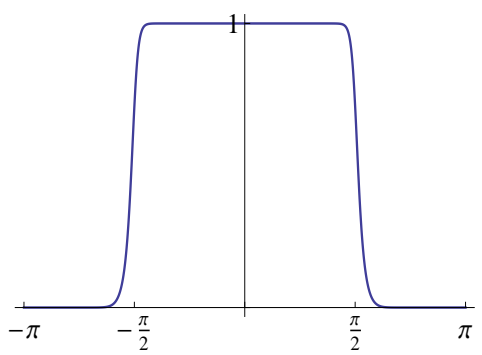

(c) $m_{D D}^{F}$

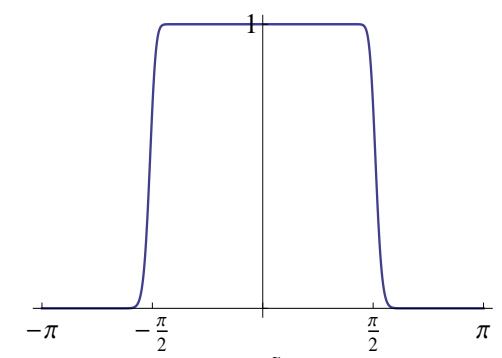

(e) $m_{D \tilde{H} F}^{\tilde{H}}$

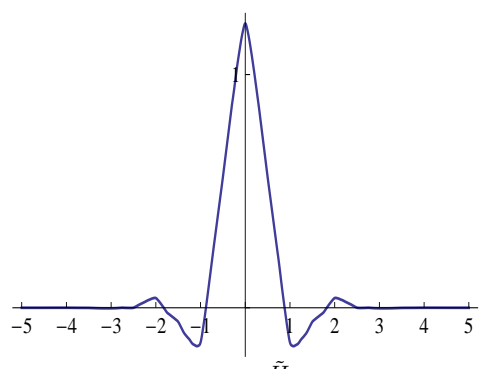

(b) $\varphi_{D}^{\tilde{H}}$

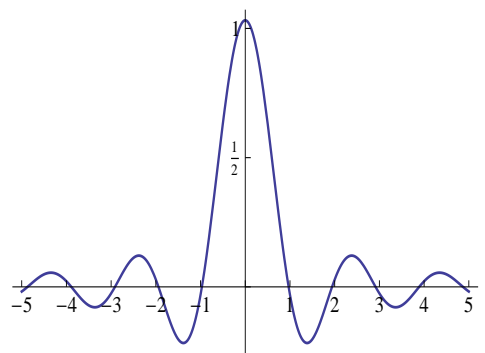

(d) $\varphi_{D D}^{F}$

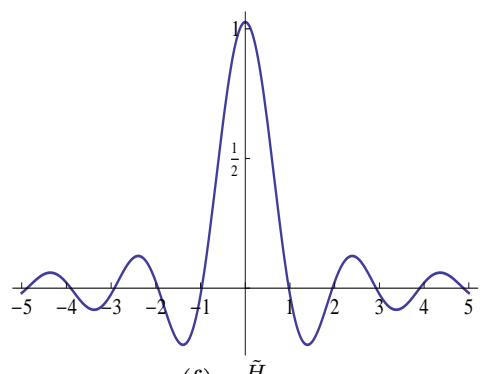

(f) $\varphi_{D \tilde{H} F}^{\tilde{H}}$

Fig. 3 Graphs of low pass filters and scaling functions.

wavelet families made by repeatedly composing $\mu$ 's would converge to the Shannon wavelet.

The Haar and Shannon scaling functions have polynomial decay of degree 1 in $\mathbf{R}_{\xi}$ and $\mathbf{R}_{x}$, respectively. Therefore, these two scaling functions would not be suitable for approximating functions which decay faster both in $\mathbf{R}_{\xi}$ and $\mathbf{R}_{x}$. Finally, let us introduce such examples of application with our scaling functions. Table 5 shows some numerical results with a relative approximation error at level 3 for $f(x)$

$$
\frac{\left\|f-P_{V_{3}} f\right\|_{L^{2}}}{\|f\|_{L^{2}}}=\sqrt{1-\frac{\left\|P_{V_{3}} f\right\|_{L^{2}}^{2}}{\|f\|_{L^{2}}^{2}}}=\sqrt{1-\frac{\sum_{k \in Z}\left|\left\langle f, \varphi_{3, k}\right\rangle\right|^{2}}{\|f\|_{L^{2}}^{2}}}
$$


Table 5 Approximation errors.

\begin{tabular}{|c|c|c|c|c|c|}
\hline \multirow{2}{*}{$f$} & \multicolumn{5}{|c|}{$\left\|f-P_{V_{3}} f\right\|_{L^{2}} /\|f\|_{L^{2}}$} \\
\cline { 2 - 6 } & Haar & Shannon & Daubechies & Meyer & $\varphi_{D}^{\tilde{H}}$ \\
\hline$\left(1-|x|^{1 / 2}\right)^{2} \chi_{[-1,1]}$ & 0.198 & 0.0951 & 0.108 & 0.0829 & 0.0584 \\
\hline$(1-|x|) \chi_{[-1,1]}$ & 0.0625 & 0.00767 & 0.0525 & 0.0110 & 0.00281 \\
\hline
\end{tabular}

where $\varphi_{3, k}(x)=2^{3 / 2} \varphi\left(2^{3} x-k\right)$. We find that $\varphi_{D}^{\tilde{H}}$ having the time-bandwidth product 0.667 gives the best approximation to the above continuous functions with compact support.

\section{Proof of Proposition 1}

We shall show the pointwise convergence in (6). For $\xi=0, \pi$, we can easily see that $m_{n}^{H}(0)=m_{\infty}^{S H}(0)=1, m_{n}^{H}(\pi)=m_{\infty}^{S H}(\pi)=0$. Since $m_{n}^{H}$ is even and $2 \pi$-periodic, it is enough to consider the two cases $0<\xi<\frac{\pi}{2}$ and $\frac{\pi}{2}<\xi<\pi$.

Define the function $f(\theta)=\frac{\pi \sin ^{2} \theta}{2 \theta}$ for $0 \leq \theta \leq \frac{\pi}{4}$. Noting that $f\left(\frac{\pi}{4}\right)=1$ and $f^{\prime}(\theta)=\frac{\pi \sin \theta(2 \theta \cos \theta-\sin \theta)}{2 \theta^{2}}>0$ for $0 \leq \theta \leq \frac{\pi}{4}$, we find that $f(\theta)$ is strictly increasing on $\left[0, \frac{\pi}{4}\right]$ and $f(\theta)<1$. For the case $0<\xi<\frac{\pi}{2}$, we have

$$
0<\Theta_{1}(\xi)<\frac{\pi}{4}
$$

and

$$
0<\Theta_{2}(\xi)\left(=\frac{\pi}{2} \sin ^{2} \Theta_{1}(\xi)\right)<\Theta_{1}(\xi)<\frac{\pi}{4} .
$$

Recursively, for $n \geq 1$, we have

$$
0<\Theta_{n}(\xi)\left(=\frac{\pi}{2} \sin ^{2} \Theta_{n-1}(\xi)\right)<\Theta_{n-1}(\xi)<\frac{\pi}{4} .
$$

Let us fix $0<\xi<\frac{\pi}{2}$. We remark that

$$
0<\Theta_{n}<\Theta_{n-1}<\cdots<\Theta_{1}<\frac{\pi}{4}
$$

since $f\left(\Theta_{n-1}\right)=\frac{\Theta_{n}}{\Theta_{n-1}}<1$. In particular, there exists a constant $a>0$ such that $f\left(\Theta_{1}\right)=\frac{\Theta_{2}}{\Theta_{1}}<a<1$. Therefore,

$$
\frac{\Theta_{n}}{\Theta_{1}}=f\left(\Theta_{n-1}\right) f\left(\Theta_{n-2}\right) \cdots f\left(\Theta_{1}\right)<f\left(\Theta_{1}\right)^{n-1}<a^{n-1} .
$$

Hence, we get $0<\Theta_{n}(\xi)<\Theta_{1} a^{n-1}<\frac{\pi}{4} a^{n-1}$ for $0<\xi<\frac{\pi}{2}$. Thus it follows that $\lim _{n \rightarrow \infty} \Theta_{n}(\xi)=0$. Consequently, we have

$$
\lim _{n \rightarrow \infty}\left|m_{n}^{H}(\xi)-m_{\infty}^{S H}(\xi)\right|=\lim _{n \rightarrow \infty}\left(1-\cos \Theta_{n}(\xi)\right)=0 .
$$


For the case $\frac{\pi}{2}<\xi<\pi$, noting that $m_{n}^{H}(\xi+\pi)=\sin \Theta_{n}(\xi)$ and $0<$ $-\xi+\pi<\frac{\pi}{2}$, we obtain $\lim _{n \rightarrow \infty} \Theta_{n}(-\xi+\pi)=0$ and also

$$
\lim _{n \rightarrow \infty}\left|m_{n}^{H}(\xi)-m_{\infty}^{S H}(\xi)\right|=\lim _{n \rightarrow \infty} m_{n}^{H}(-\xi)=\lim _{n \rightarrow \infty} \sin \Theta_{n}(-\xi+\pi)=0 .
$$

Since $\Theta_{n}\left(\frac{\pi}{2}\right)=\frac{\pi}{4}$ and (10), we also obtain (5).

\section{Proof of Theorem 1}

It is sufficient to give a proof only for the scaling functions. First, we shall prove that for $1 \leq p<\infty$,

$$
\lim _{n \rightarrow \infty}\left\|\hat{\varphi}_{n}^{H}-\hat{\varphi}_{\infty}^{S H}\right\|_{L^{p}(\mathbf{R})}=0 .
$$

Let us fix $\xi \in \mathbf{R}$. Then, for any $\varepsilon>0$, there exists $J>0$ such that

$$
\left|\hat{\varphi}_{n}^{H}(\xi)-\prod_{j=1}^{J} m_{n}^{H}\left(2^{-j} \xi\right)\right|<\frac{\varepsilon}{3} \text { and }\left|\hat{\varphi}_{\infty}^{S H}(\xi)-\prod_{j=1}^{J} m_{\infty}^{S H}\left(2^{-j} \xi\right)\right|<\frac{\varepsilon}{3} .
$$

For a sufficiently large $N=N(J)>0$, Proposition 1 gives that, for $\xi \in$ $\mathbf{R} \backslash \bigcup_{j=1}^{J} 2^{j}\left\{\pi \mathbf{Z}+\frac{\pi}{2}\right\}$,

$$
\left|\prod_{j=1}^{J} m_{N}^{H}\left(2^{-j} \xi\right)-\prod_{j=1}^{J} m_{\infty}^{S H}\left(2^{-j} \xi\right)\right|<\frac{\varepsilon}{3} .
$$

Thus it follows that for $\xi \in \mathbf{R} \backslash \bigcup_{j=1}^{J} 2^{j}\left\{\pi \mathbf{Z}+\frac{\pi}{2}\right\}$

$$
\begin{aligned}
& \left|\hat{\varphi}_{N}^{H}(\xi)-\hat{\varphi}_{\infty}^{S H}(\xi)\right| \\
\leq & \left|\hat{\varphi}_{N}^{H}(\xi)-\prod_{j=1}^{J} m_{N}^{H}\left(2^{-j} \xi\right)\right|+\left|\prod_{j=1}^{J} m_{N}^{H}\left(2^{-j} \xi\right)-\prod_{j=1}^{J} m_{\infty}^{S H}\left(2^{-j} \xi\right)\right| \\
& \quad+\left|\prod_{j=1}^{J} m_{\infty}^{S H}\left(2^{-j} \xi\right)-\hat{\varphi}_{\infty}^{S H}(\xi)\right| \\
& <\varepsilon .
\end{aligned}
$$

This implies that for almost all $\xi \in \mathbf{R}$,

$$
\lim _{n \rightarrow \infty}\left|\hat{\varphi}_{n}^{H}(\xi)-\hat{\varphi}_{\infty}^{S H}(\xi)\right|=0 .
$$

From the proof of Theorem 2, we will find that $\varphi_{n}^{H}(x)$ is smooth and $\left|\hat{\varphi}_{n}^{H}(\xi)\right|^{p}$ is dominated by some integrable function for a sufficiently large $n=n(p)>0$. Therefore, the dominated convergence theorem proves (11).

Let us consider (11) especially for $1<p \leq 2$. Taking $2 \leq q<\infty$ such that $\frac{1}{p}+\frac{1}{q}=1$, by the Hausdorff-Young inequality, we get

$$
\lim _{n \rightarrow \infty}\left\|\varphi_{n}^{H}-\varphi_{\infty}^{S H}\right\|_{L^{q}(\mathbf{R})}=0 .
$$




\section{Proof of Theorem 2}

In order to know the decays and regularities, we shall rewrite (4) as follows:

Lemma 1 For $n \geq 2, m_{n}^{H}(\xi)$ can be rewritten as

$$
m_{n}^{H}(\xi)=\left(\frac{\pi}{2}\right)^{2^{n-1}-1} \cos ^{2^{n-1}} \frac{\xi}{2} \prod_{k=1}^{n-1} \operatorname{sinc}^{2^{k-1}}\left(\frac{\pi}{2} \cos ^{2} \Theta_{n-k}(\xi)\right)
$$

Proof For $n=2$, we obtain

$$
\begin{aligned}
m_{2}^{H}(\xi) & =\cos \Theta_{2}(\xi)=\cos \left(\frac{\pi}{2} \sin ^{2} \frac{\xi}{2}\right)=\sin \left(\frac{\pi}{2} \cos ^{2} \frac{\xi}{2}\right) \\
& =\frac{\pi}{2} \cos ^{2} \frac{\xi}{2} \operatorname{sinc}\left(\frac{\pi}{2} \cos ^{2} \frac{\xi}{2}\right) .
\end{aligned}
$$

We shall assume that (12) holds for $n=N$ and show the $n=N+1$ case. Noting that $\Theta_{N+1}(\xi)=\Theta_{N}\left(\pi \sin ^{2} \frac{\xi}{2}\right)$ and $\cos \left(\frac{\pi}{2} \sin ^{2} \frac{\xi}{2}\right)=\sin \left(\frac{\pi}{2} \cos ^{2} \frac{\xi}{2}\right)$, we have

$$
\begin{aligned}
& m_{N+1}^{H}(\xi) \\
= & \cos \Theta_{N}\left(\pi \sin ^{2} \frac{\xi}{2}\right)=m_{N}^{H}\left(\pi \sin ^{2} \frac{\xi}{2}\right) \\
= & \left(\frac{\pi}{2}\right)^{2^{N-1}-1} \cos ^{2^{N-1}}\left(\frac{\pi}{2} \sin ^{2} \frac{\xi}{2}\right) \prod_{k=1}^{N-1} \operatorname{sinc}^{2^{k-1}}\left(\frac{\pi}{2} \cos ^{2} \Theta_{N-k}\left(\pi \sin ^{2} \frac{\xi}{2}\right)\right) \\
= & \left(\frac{\pi}{2}\right)^{2^{N-1}-1} \sin ^{2^{N-1}}\left(\frac{\pi}{2} \cos ^{2} \frac{\xi}{2}\right) \prod_{k=1}^{N-1} \operatorname{sinc}^{2^{k-1}}\left(\frac{\pi}{2} \cos ^{2} \Theta_{N+1-k}(\xi)\right) \\
= & \left(\frac{\pi}{2}\right)^{2^{N-1}-1}\left(\frac{\pi}{2} \cos ^{2} \frac{\xi}{2}\right)^{2^{N-1}} \operatorname{sinc}^{2^{N-1}}\left(\frac{\pi}{2} \cos ^{2} \frac{\xi}{2}\right) \\
= & \left(\frac{\pi}{2}\right)^{2^{N}-1} \cos ^{2^{N}} \frac{\xi}{2} \prod_{k=1}^{N-1} \operatorname{sinc}^{2^{k-1}}\left(\frac{\pi}{2} \cos ^{2} \Theta^{2^{k-1}}\left(\frac{\pi}{2} \cos ^{2} \Theta_{N+1-k}(\xi)\right)\right.
\end{aligned}
$$

This proves (12). 
From Lemma 1, it follows that for $n \geq 2$,

$$
\begin{aligned}
\hat{\varphi}_{n}^{H}(\xi) & =\prod_{j=1}^{\infty} m_{n}^{H}\left(\frac{\xi}{2^{j}}\right) \\
& =\prod_{j=1}^{\infty}\left\{\left(\frac{\pi}{2}\right)^{2^{n-1}-1} \cos ^{2^{n-1}}\left(\frac{\xi}{2^{j+1}}\right) \prod_{k=1}^{n-1} \operatorname{sinc}^{2^{k-1}}\left(\frac{\pi}{2} \cos ^{2} \Theta_{n-k}\left(\frac{\xi}{2^{j}}\right)\right)\right\} \\
& =\operatorname{sinc}^{2^{n-1}}\left(\frac{\xi}{2}\right) \prod_{j=1}^{\infty} L_{n}^{H}\left(\frac{\xi}{2^{j}}\right)
\end{aligned}
$$

where

$$
L_{n}^{H}(\xi)=\left(\frac{\pi}{2}\right)^{2^{n-1}-1} \prod_{k=1}^{n-1} \operatorname{sinc}^{2^{k-1}}\left(\frac{\pi}{2} \cos ^{2} \Theta_{n-k}(\xi)\right) .
$$

By $L_{n}^{H}(0)=1$ and continuity, there exists $C>0$ such that

$$
\left|L_{n}^{H}(\xi+i \eta)\right| \leq 1+C|\xi+i \eta| \text { in some neighborhood of the origin. }
$$

While, since $\left|L_{n}^{H}(\xi)\right| \leq\left(\frac{\pi}{2}\right)^{2^{n-1}-1}$ for $\xi \in \mathbf{R}$, for all $\varepsilon>0$ there exists $\delta_{\xi}>0$ such that for all $\eta$ with $0<|\eta|<\delta_{\xi}$, we have

$$
\left|L_{n}^{H}(\xi+i \eta)\right| \leq\left(\frac{\pi}{2}\right)^{2^{n-1}-1+\varepsilon} .
$$

We note that $L_{n}^{H}(\xi)$ is $2 \pi$-periodic and $\sup _{\xi \in \mathbf{R}} \delta_{\xi}=\max _{0 \leq \xi \leq 2 \pi} \delta_{\xi}>0$. So, we can take $\delta=\max _{0 \leq \xi \leq 2 \pi} \delta_{\xi}>0$ which is independent of $\xi$. For an arbitrary fixed $J \geq 1$, taking $\eta$ such that $\max _{1 \leq j \leq J}\left|\frac{\eta}{2^{j}}\right|<\delta$, i.e., $|\eta|<2 \delta$, by (14) and (15), we get for $2^{J} \leq|\xi+i \eta| \leq 2^{J+1}$

$$
\begin{aligned}
\left|\hat{\varphi}_{n}^{H}(\xi+i \eta)\right| & =\left|\operatorname{sinc}\left(\frac{\xi+i \eta}{2}\right)\right|^{2^{n-1}} \prod_{j=1}^{J}\left|L_{n}^{H}\left(\frac{\xi+i \eta}{2^{j}}\right)\right| \prod_{j=J+1}^{\infty}\left|L_{n}^{H}\left(\frac{\xi+i \eta}{2^{j}}\right)\right| \\
& \leq\left|\frac{\sin \left(\frac{\xi+i \eta}{2}\right)}{\left(\frac{\xi+i \eta}{2}\right)}\right|^{2^{n-1}}\left(\frac{\pi}{2}\right)^{J\left(2^{n-1}-1+\varepsilon\right)} \prod_{j=J+1}^{\infty}\left\{1+C\left|\frac{\xi+i \eta}{2^{j}}\right|\right\} \\
& \leq \frac{C_{n, \eta}}{|\xi+i \eta|^{2^{n-1}}+1} 2^{J\left(2^{n-1}-1+\varepsilon\right) \log _{2} \frac{\pi}{2}} \prod_{j=J+1}^{\infty} \exp \left\{C\left|\frac{\xi+i \eta}{2^{j}}\right|\right\} \\
& \leq \frac{C_{n, \eta}}{|\xi+i \eta|^{2^{n-1}}+1}|\xi+i \eta|^{\left(2^{n-1}-1+\varepsilon\right) \log _{2} \frac{\pi}{2}} \exp \left\{\sum_{j=0}^{\infty} \frac{C}{2^{j}}\right\} \\
& \leq C_{n, \eta} e^{2 C}(|\xi|+|\eta|+1)^{q_{n, \varepsilon}},
\end{aligned}
$$

where $q_{n, \varepsilon}=\left(2^{n-1}-1+\varepsilon\right) \log _{2} \frac{\pi}{2}-2^{n-1}$ and $C_{n, \eta}=2^{2^{n-1}}(\cosh \eta)^{2^{n-2}}$. For $|\xi+i \eta| \leq 1$ we easily see that $\left|\hat{\varphi}_{n}^{H}(\xi+i \eta)\right| \leq C^{\prime}$. Thus, it follows that for $\xi \in \mathbf{R}$ and $|\eta|<2 \delta$

$$
\left|\hat{\varphi}_{n}^{H}(\xi+i \eta)\right| \leq M_{n, \eta}(|\xi|+|\eta|+1)^{q_{n, \varepsilon}},
$$


where $M_{n, \eta}=C_{n, \eta} e^{2 C}+C^{\prime}$. The exponent $q_{n, \varepsilon}$ becomes negative for a sufficiently small $\varepsilon>0$. Thus, by the Paley-Wiener theorem we conclude the following two facts:

- $\varphi_{n}^{H}$ has exponential decay, since $\hat{\varphi}_{n}^{H}$ is analytic in a strip $\{\xi+i \eta:|\eta|<\tilde{\delta}\}$ for some $\tilde{\delta}$.

- $\varphi_{n}^{H}$ belongs to $C^{\alpha_{n}}\left(\mathbf{R}_{x}\right)$, where $\alpha_{n}=\left(2^{n-1}-1\right)\left(1-\log _{2} \frac{\pi}{2}\right)$ (estimate (16) with $\eta=0$ ) which is increasing in the parameter $n \geq 2$.

Remark 6 In the above computations, we took the constant $C_{n, \eta}>0$ such that

$$
C_{n, \eta} \geq \sup _{\xi}\left|2 \sin \left(\frac{\xi+i \eta}{2}\right)\right|^{2^{n-1}} \geq\left|2 \cosh \left(\frac{\eta}{2}\right)\right|^{2^{n-1}}\left(\geq 2^{2^{n-1}}\right) .
$$

Hence, noting that $q_{n, \varepsilon}>-2^{n-1}$, we would get

$$
\begin{aligned}
C_{n, \eta} e^{2 C}(|\xi|+|\eta|+1)^{q_{n, \varepsilon}} & \geq C\left|2 \cosh \left(\frac{\eta}{2}\right)\right|^{2^{n-1}}(|\xi|+|\eta|+1)^{q_{n, \varepsilon}} \\
& \geq C\left|\frac{2 \cosh \left(\frac{\eta}{2}\right)}{|\eta|+1}\right|^{2^{n-1}} \rightarrow \infty \quad(n \rightarrow \infty),
\end{aligned}
$$

here we used $\left|\frac{2 \cosh \left(\frac{\eta}{2}\right)}{|\eta|+1}\right|>1$. This means that $\varphi_{n}^{H}(x)$ fails to decay exponentially as $n$ tends to infinity. While, with small changes, we could also obtain a rough estimate for $\eta=0$

$$
\left|\hat{\varphi}_{n}^{H}(\xi)\right| \leq C 2^{-\lambda J 2^{n-1}} \quad \text { for } 2^{J} \leq|\xi| \leq 2^{J+1},
$$

taking sufficiently small $\lambda>0$ and sufficiently large $J \geq 1$. Hence, it follows that

$$
\left|\hat{\varphi}_{n}^{H}(\xi)\right| \leq C(|\xi|+1)^{-\lambda 2^{n-1}}
$$

where $C>0$ is independent of $n$. This means that as $n$ tends to infinity, $\varphi_{n}^{H}(x)$ becomes more regular.

Now we shall improve the value of $\alpha_{n}$. For this purpose, we need the following lemma (see [2] ):

Lemma 2 Let $M \geq 1$. Suppose that $[-\pi, \pi]=D_{1} \cup D_{2} \cup \cdots \cup D_{M}$, and there exists $q>0$ so that

$$
\begin{array}{ll}
\left|L_{n}^{H}(\xi)\right| \leq q & \text { for } \quad \xi \in D_{1}, \\
\left|L_{n}^{H}(\xi) L_{n}^{H}(2 \xi)\right| \leq q^{2} & \text { for } \quad \xi \in D_{2}, \\
\quad \vdots & \\
\left|L_{n}^{H}(\xi) L_{n}^{H}(2 \xi) \cdots L_{n}^{H}\left(2^{M-1} \xi\right)\right| \leq q^{M} & \text { for } \quad \xi \in D_{M} .
\end{array}
$$

Then, it holds that $\left|\prod_{j=1}^{\infty} L\left(2^{-j} \xi\right)\right| \leq C(1+|\xi|)^{K}$, where $K=\log q / \log 2$. 
For $L_{2}^{H}(\xi)$, with $M=2$ and $q=L_{2}^{H}\left(\frac{2 \pi}{3}\right)$ we can prove the following:

Lemma 3

$$
\begin{array}{ll}
\left|L_{2}^{H}(\xi)\right| \leq L_{2}^{H}\left(\frac{2 \pi}{3}\right) & \text { for } \quad 0<|\xi|<\frac{2 \pi}{3}, \\
\left|L_{2}^{H}(\xi) L_{2}^{H}(2 \xi)\right| \leq L_{2}^{H}\left(\frac{2 \pi}{3}\right)^{2} & \text { for } \quad \frac{2 \pi}{3}<|\xi|<\pi .
\end{array}
$$

Proof Since $L_{2}^{H}(\xi)=\frac{\pi}{2} \operatorname{sinc}\left(\frac{\pi}{2} \cos ^{2} \frac{\xi}{2}\right)$ is increasing on $\left[0, \frac{2 \pi}{3}\right]$, it is sufficient to show (19).

Let us define $g(\xi)=\tilde{g}(\cos \xi)$, where $\tilde{g}(\omega)=\operatorname{sinc}\left(\frac{\pi}{2} \frac{1+\omega^{2}}{2}\right) \operatorname{sinc}\left(\frac{\pi}{2} \omega^{2}\right)$. We remark that $-1 \leq \omega \leq-\frac{1}{2}, 0 \leq \frac{\pi}{2} \frac{1+\omega^{2}}{2} \leq \frac{\pi}{8}$, and $\frac{\pi}{8} \leq \frac{\pi}{2} \omega^{2} \leq \frac{\pi}{2}$ for $\frac{2 \pi}{3} \leq \xi \leq \pi$.

By a simple calculation, we get $\left|L_{2}^{H}(\xi) L_{2}^{H}(2 \xi)\right|=\left(\frac{\pi}{2}\right)^{2} g(\xi)$ and

$$
\begin{aligned}
\tilde{g}^{\prime}(\omega) & =\frac{\cos \left(\frac{\pi}{2} \frac{1+\omega}{2}\right)-\operatorname{sinc}\left(\frac{\pi}{2} \frac{1+\omega}{2}\right)}{\frac{\pi}{2} \frac{1+\omega}{2}} \frac{\pi}{4} \operatorname{sinc}\left(\frac{\pi}{2} \omega^{2}\right) \\
& +\frac{\cos \left(\frac{\pi}{2} \omega^{2}\right)-\operatorname{sinc}\left(\frac{\pi}{2} \omega^{2}\right)}{\frac{\pi}{2} \omega^{2}} \pi \omega \operatorname{sinc}\left(\frac{\pi}{2} \frac{1+\omega}{2}\right) \\
& =h\left(\frac{\pi}{2} \frac{1+\omega}{2}\right) \frac{\pi}{4} \operatorname{sinc}\left(\frac{\pi}{2} \omega^{2}\right) \operatorname{sinc}\left(\frac{\pi}{2} \frac{1+\omega}{2}\right) \\
& +h\left(\frac{\pi}{2} \omega^{2}\right) \pi \omega \operatorname{sinc}\left(\frac{\pi}{2} \omega^{2}\right) \operatorname{sinc}\left(\frac{\pi}{2} \frac{1+\omega}{2}\right),
\end{aligned}
$$

where $h(\xi)=\frac{\cos \xi-\operatorname{sinc} \xi}{\xi \operatorname{sinc} \xi}=\frac{\xi \cos \xi-\sin \xi}{\xi \sin \xi}$. Since $h^{\prime}(\xi)=\frac{1}{\xi^{2}}-\frac{1}{\sin ^{2} \xi}<0$ for $0<\xi<$ $\frac{\pi}{2}, h(\xi)$ is decreasing on $\left[0, \frac{\pi}{2}\right]$, and by l'Hôpital's rule, $h(0)=\lim _{\xi \rightarrow 0} h(\xi)=0$. Hence, we obtain

$$
0 \geq h\left(\frac{\pi}{2} \frac{1+\omega}{2}\right) \geq h\left(\frac{\pi}{2} \omega^{2}\right) .
$$

Since $0<\frac{\pi}{4} \leq-\pi \omega$ for $-1 \leq \omega \leq-\frac{1}{2}$, by (20) it holds that

$$
h\left(\frac{\pi}{2} \frac{1+\omega}{2}\right) \frac{\pi}{4} \geq-h\left(\frac{\pi}{2} \omega^{2}\right) \pi \omega .
$$

Multiplying (21) by $\operatorname{sinc}\left(\frac{\pi}{2} \omega^{2}\right) \operatorname{sinc}\left(\frac{\pi}{2} \frac{1+\omega}{2}\right)>0$ gives $\tilde{g}^{\prime}(\omega) \geq 0$ for $-1 \leq \omega \leq$ $-\frac{1}{2}$, and we have for $\frac{2 \pi}{3} \leq \xi \leq \pi$,

$$
\frac{d}{d \xi}\left|L_{2}^{H}(\xi) L_{2}^{H}(2 \xi)\right|=\left(\frac{\pi}{2}\right)^{2} \frac{d}{d \omega} \tilde{g}(\omega) \frac{d \omega}{d \xi}=-\left(\frac{\pi}{2}\right)^{2} \tilde{g}^{\prime}(\omega) \sin \xi \leq 0 .
$$

Therefore, $\left|L_{2}^{H}(\xi) L_{2}^{H}(2 \xi)\right|$ is decreasing on $\left[\frac{2 \pi}{3}, \pi\right]$. Since $L_{2}^{H}\left(\frac{4 \pi}{3}\right)=L_{2}^{H}\left(\frac{2 \pi}{3}\right)$, it follows that $\left|L_{2}^{H}(\xi) L_{2}^{H}(2 \xi)\right| \leq\left|L_{2}^{H}\left(\frac{2 \pi}{3}\right) L_{2}^{H}\left(\frac{4 \pi}{3}\right)\right|=L_{2}^{H}\left(\frac{2 \pi}{3}\right)^{2}=2.343$ for $\left[\frac{2 \pi}{3}, \pi\right]$. 
Thus, from Lemmas 2 and 3, it follows that

$$
\hat{\varphi}_{2}^{H}(\xi) \leq C(1+|\xi|)^{-2+\log L_{2}^{H}(2 \pi / 3) / \log 2},
$$

and we have $\alpha_{2}=1-\log L_{2}^{H}(2 \pi / 3) / \log 2=0.386$. For $3 \leq n \leq 6$, by numerical computations, one can see that (19) also holds for $3 \leq n \leq 6$.

Table 1 gives the value of $\alpha_{n}$ for $2 \leq n \leq 6$.

\section{Proof of Proposition 2}

(i) Since $m$ is a low pass filter with real Fourier coefficients, we find that $|m(\xi)|\left(=\sqrt{\sum_{k \in Z} a_{k} e^{-i k \xi} \sum_{k^{\prime} \in Z} a_{k^{\prime}} e^{i k^{\prime} \xi}}\right)$ is an even function. Noting that $\Theta_{n}(\xi+\pi)=\frac{\pi}{2}-\Theta_{n}(\xi)$, we have

$$
\begin{aligned}
\left|m_{n}(\xi+\pi)\right| & =\left|m\left(2 \Theta_{n}(\xi+\pi)\right)\right|=\left|m\left(\pi-2 \Theta_{n}(\xi)\right)\right| \\
& =\left|m\left(2 \Theta_{n}(\xi)-\pi\right)\right|=\left|m\left(2 \Theta_{n}(\xi)+\pi\right)\right| .
\end{aligned}
$$

Therefore, it follows that

$$
\left|m_{n}(\xi)\right|^{2}+\left|m_{n}(\xi+\pi)\right|^{2}=\left|m\left(2 \Theta_{n}(\xi)\right)\right|^{2}+\left|m\left(2 \Theta_{n}(\xi)+\pi\right)\right|^{2}=1
$$

and $m_{n}(0)=m\left(2 \Theta_{n}(0)\right)=m(0)=1$.

(ii) By the assumptions, $m(\xi)$ can be factored as $m(\xi)=\cos \frac{\xi}{2} L(\xi)$, where $L(\xi)$ is the $2 \pi$-periodic continuous function (see [2]). Hence, we have

$$
\begin{aligned}
\hat{\varphi}_{n}(\xi) & =\prod_{j=1}^{\infty} \cos \Theta_{n}\left(2^{-j} \xi\right) \prod_{j=1}^{\infty} L\left(2 \Theta_{n}\left(2^{-j} \xi\right)\right) \\
& =\hat{\varphi}_{n}^{H}(\xi) \prod_{j=1}^{\infty} L\left(2 \Theta_{n}\left(2^{-j} \xi\right)\right) .
\end{aligned}
$$

Moreover, since $L(\xi)$ is a $2 \pi$-periodic continuous function, there exists a constant $C>0$ such that $\sup _{\xi}\left|L\left(\Theta_{n}\left(2^{-j} \xi\right)\right)\right|<2^{C}$. Here we remark that $C$ is independent of $j \geq 1$ and $n \geq 2$, since $0 \leq \Theta_{n} \leq \frac{\pi}{2}$. Then, in a similar manner as in $\S 6$, we find

$$
\prod_{j=1}^{\infty} L\left(2 \Theta_{n}\left(2^{-j} \xi\right)\right)<C^{\prime}(1+|\xi|)^{C-\varepsilon} .
$$

Thus, from the proof of Theorem 2, it follows that

$$
\left|\hat{\varphi}_{n}(\xi)\right| \leq C^{\prime \prime}(1+|\xi|)^{q_{n, \varepsilon}+C-\varepsilon} .
$$

Consequently, $\varphi_{n} \in C^{\gamma_{n}}$, where $\gamma_{n}=\max \left\{0, \alpha_{n}-C\right\}$ is increasing in the parameter $n$. 


\section{Sketch of Proofs of Theorems 3 and 4}

We shall devote ourselves only to the case of the scaling functions. To prove Theorem 3, we need the corresponding result to Proposition 1 for the Franklin wavelet,

$$
\lim _{n \rightarrow \infty} m_{n}^{F}(\xi)=m_{\infty}^{S H}(\xi) \text { for } \xi \in \mathbf{R} \backslash\left\{\pi \mathbf{Z}+\frac{\pi}{2}\right\} .
$$

For the case $0 \leq \xi<\frac{\pi}{2}$, by $\lim _{n \rightarrow \infty} \Theta_{n}(\xi)=0$, we get

$$
\begin{aligned}
\lim _{n \rightarrow \infty}\left|m_{n}^{F}(\xi)-m_{\infty}^{S H}(\xi)\right| & =\lim _{n \rightarrow \infty}\left(1-\cos ^{2} \Theta_{n}(\xi) \sqrt{\frac{2+\cos \left(2 \Theta_{n}(\xi)\right)}{2+\cos \left(4 \Theta_{n}(\xi)\right)}}\right) \\
& =1-1 \sqrt{\frac{3}{3}}=0 .
\end{aligned}
$$

For the case $\frac{\pi}{2}<\xi<\pi$, we have

$$
\begin{aligned}
\left|m_{n}^{F}(\xi)-m_{\infty}^{S H}(\xi)\right| & =\cos ^{2} \Theta_{n}(\xi) \sqrt{\frac{2+\cos \left(2 \Theta_{n}(\xi)\right)}{2+\cos \left(4 \Theta_{n}(\xi)\right)}} \\
& \leq \sqrt{3} \cos ^{2} \Theta_{n}(\xi)=\sqrt{3} m_{n}^{H}(\xi)^{2}
\end{aligned}
$$

Hence, by Proposition 1, we obtain $\lim _{n \rightarrow \infty}\left|m_{n}^{F}(\xi)-m_{\infty}^{S H}(\xi)\right|=0$. Finally, for the case $\xi=\pi$, we can easily see that $m_{n}^{F}(\xi)=m_{\infty}^{S H}(\xi)=1$. Now, by the same method as used in $\S 5$, we can prove Theorem 3.

Moreover, we can also verify that

$$
\hat{\varphi}_{n}^{F}(\xi)=\operatorname{sinc}^{2}\left(\frac{\xi}{2}\right) \prod_{j=1}^{\infty} L_{n}^{F}\left(\frac{\xi}{2^{j}}\right)
$$

where

$$
L_{n}^{F}(\xi)=\left(\frac{\pi}{2}\right)^{2\left(2^{n-1}-1\right)} \sqrt{\frac{2+\cos \left(2 \Theta_{n}(\xi)\right)}{2+\cos \left(4 \Theta_{n}(\xi)\right)}} \prod_{k=1}^{n-1} \operatorname{sinc}^{2^{k}}\left(\frac{\pi}{2} \cos ^{2} \Theta_{n-k}(\xi)\right) .
$$

Furthermore, it follows that

$$
\begin{aligned}
\sup _{\xi}\left|L_{n}^{F}(\xi)\right| & \leq \sup _{0 \leq \xi \leq \pi}\left(\frac{\pi}{2}\right)^{2\left(2^{n-1}-1\right)} \sqrt{\frac{2+\cos (\xi)}{2+\cos (2 \xi)}} \\
& =2^{2^{n-\left(2^{n}-1-2\left(2^{n-1}-1\right) \log _{2} \frac{\pi}{2}-\log _{2} 1.4355\right)-1}} .
\end{aligned}
$$

Here we used $\sup _{0 \leq \xi \leq \pi} \sqrt{\frac{2+\cos (\xi)}{2+\cos (2 \xi)}}=1.4355$. Then, in a similar manner as in $\S 6$, we find that $\varphi_{n}^{F}(\xi)$ has exponential decay and belongs to $C^{\beta_{n}}\left(\mathbf{R}_{x}\right)$, where $\beta_{n}=2\left(2^{n-1}-1\right)\left(1-\log _{2} \frac{\pi}{2}\right)+1-\log _{2} 1.4355$.

Table 4 gives the value of $\beta_{n}$ for $2 \leq n \leq 6$.

Acknowledgements The authors would like to thank the referees for valuable suggestions. 


\section{References}

1. Battle, G.: A block spin construction of ondelettes. I. Lemarié functions, Comm. Math. Phys., 110, 601-615 (1987)

2. Daubechies, I.: Ten lectures on wavelets, CBMS-NSF Regional Conference Series in Applied Mathematics, 61, SIAM, Philadelphia, PA, (1992)

3. Fukuda, N., Kinoshita, T.: On non-symmetric orthogonal spline wavelets, to appear in Southeast Asian Bulletin of Mathematics.

4. Fukuda, N., Kinoshita, T.: On the new family of wavelets interpolating to the Shannon wavelet, JSIAM Letters, 3, 33-36, (2011)

5. Hernández, E., Weiss, G.: A first course on wavelets, CRC Press, Boca Raton, FL, (1996)

6. Kateb, D., Lemarié-Rieusset, P.G.: Asymptotic behavior of the Daubechies filters, Appl. Comput. Harmon. Anal. 2, 398-399 (1995)

7. Kim, H. O., Kim, R. Y., Ku, J. S.: On asymptotic behavior of Battle-Lemarié scaling functions and wavelets, Appl. Math. Lett., 20, 376-381 (2007)

8. Krantz, S.G., Parks, H.R.: A Primer of Real Analytic Functions, Birkhäuser, BostonBasel-Berlin, 2nd edition (2002)

9. Lemarié, P.G.: Ondelettes à localisation exponentielles, J. Math. pures et appl., 67, No. 3, 227-236 (1988)

10. Schoenberg, I.J.: Cardinal interpolation and spline functions, J. Approximation Theory, 2, 167-206 (1969)

11. Strömberg, J.O.: A modified Franklin system and higher-order spline systems on $\mathbf{R}^{n}$ as unconditional basis for Hardy spaces, Proc. Conference on Harmonic Analysis in honor of Antoni Zygmund, 475-493 (1983)

12. Unser, M., Aldroubi, A., Eden, M.: On the asymptotic convergence of $B$-spline wavelets to Gabor functions, IEEE Trans. Inform. Theory 38, No. 2, 864-872 (1992) 